IRSTI 27.35.57

\title{
Asymptotic behavior of numerical solutions of the Schrodinger equation
}

\author{
F.M. Pen'kov ${ }^{1}$ and P.M. Krassovitskiy ${ }^{1,2}$ \\ ${ }^{1}$ Institute of nuclear physics, 1, Ibragimov Str., 050032, Almaty, Kazakhstan \\ ${ }^{2}$ Join institute for nuclear research, 6, Joliot-Curie Str., 141980, Dubna, Russia \\ e-mail:fmp56@mail.ru
}

\begin{abstract}
Many problems of numerically solving the Schrodinger equation require that we choose asymptotic distances many times greater than the characteristic size of the region of interaction. The problems of resonance diffraction for composite particles or the problem of nucleon scattering by nonspherical atomic nuclei are examples of the need to use a large spatial domain for calculations. If the solution to onedimensional equations can be immediately chosen in a form that preserves unitarity, the invariance of probability (in the form of, e.g., fulfilling an optical theorem) is a real problem for two-dimensional equations. An addition that does not exceed the discretization error and ensures a high degree of unitarity is proposed as a result of studying the properties of a discrete two-dimensional equation.

The problem for scattering of rigid molecules by the disks was successfully solved using an improved sampling scheme that provides the correct asymptotic behavior. Corresponding diffraction scattering curves are of a pronounced resonance nature.
\end{abstract}

Key words: Numerov's method, resonance diffraction, Babine's principle.

PACS numbers 2.60.- $\mathrm{x}, 03.65 . \mathrm{Nk}, 34.50 .-\mathrm{s}$

\section{Introduction}

In numerically solving problems whose mathematical notation is expressed in terms of the two-dimensional Schrodinger equation, there is the problem of a loss of accuracy for finite difference schemes. It is associated with the need to travel long distances relative to the characteristic size of the potential range. Such situations arise in the scattering of slow particles at long scattering distances or with the scattering of fast particles with pronounced diffraction when we need to go beyond the region of the diffraction shadow in the calculations.

The problems of resonance diffraction for composite particles $[1,2]$ or the problem of nucleon scattering by nonspherical atomic nuclei are examples of the need to use a large spatial domain for calculations. Expansion in spherical functions in this case loses both physical and mathematical meaning because of the non conservation of the angular momentum in the scattering process. The standard approach, which uses amplitude notation in an explicitly unitary form, thus becomes an inoperative tool. We can, of course, choose a solving scheme based on some averaging of the nonspherical potential (by, e.g., means of folding [3]) and leaving the error of the means of solving without any possibility of analysis. This problem arises not only in the two examples given above, and it is solved in different ways for specific cases in theoretical physics, nanostructure physics, and related fields of chemistry, medicine, and atomic interferometry [4-8].

\section{Material and methods}

Let us write the Schrodinger equation in a twodimensional axially symmetric case for a scattering problem (here and below, we use a system of units in which the Planck constant and the Boltzmann constant are 1 in analogy with [4]) 


$$
\begin{gathered}
\Delta \Psi(\vec{r})-V(\vec{r}) \Psi(\vec{r})=-k^{2} \Psi(\vec{r}), \\
\Delta=\frac{\partial^{2}}{\partial r^{2}}+\frac{2}{r} \frac{\partial}{\partial r}+\frac{1}{r^{2}} \frac{1}{\sin (\vartheta)} \frac{\partial}{\partial \vartheta} \sin (\vartheta) \frac{\partial}{\partial \vartheta}
\end{gathered}
$$

Here, $k^{2}=2 m E$ is a wave parameter, $E$ is the energy of the system, and $\Psi(\vec{r})=\Psi(r, \vartheta)$ is the wave function. Note that the energy in the selected system of units ismeasured in ones that are inverse to the square of thelength. The potential is limited in some area $V=0$ with $r>r_{V}$. The asymptotic form of the wave functionin a region greater than some asymptotic radius $r_{a s}: r>r_{a s}>>r_{V}$,

$$
\Psi(\vec{r}) \rightarrow e^{i \vec{k} \vec{r}}+f(\vartheta) \frac{e^{i k r}}{r}\left(1+O\left(\frac{1}{r}\right)\right),
$$

determines scattering amplitude $f(\vartheta)$, which obeys optical theorem [9]

$$
\sigma=2 \pi \int_{0}^{\pi}|f(\vartheta)|^{2} \sin (\vartheta) d \vartheta=\frac{4 \pi}{k} \operatorname{Im} f(0)
$$

where $\sigma$ is the total cross section. This ratio (the conditionfor invariance of probability) can be used as acriterion of accuracy for the numerical solution to theproblem. We must choose a sufficiently high value of parameter $r_{\mathrm{as}}$ that determines the region of the asymptoticsolution in the numerical solution to the scatteringproblem. Asymptotic distances several hundred unitsof the problem's length for characteristic values $r_{V}=1-3$ and $k \leq 30$ must extend beyond shadow region $r_{a s}>r_{V}\left(k r_{V}\right)$ in the problem of the quantum scattering of particles on a nonspherical potential [1].

The limiting accuracy of calculations for such distancesis determined by the ignored asymptotic term (i.e., it can be a fraction of a percent). The form of potential $V(\vec{r})$ requires the use of asmall parameter for the sampling step. This ensuresthe accuracy of the solution, but considerably extendsthe estimated time. Two sampling steps are used toadvance the calculations for continuing quantum scatteringstudies on nonspherical potentials [1,2]. For $r \leq r_{s} \approx r_{V}$, we choose fairly small step $\Delta r=0.001$, which guarantees sufficient accuracy $O\left((\Delta r)^{2}\right)$ in the range of potentials, and step $\quad \Delta r_{2}=2^{n} \Delta r$ with $\quad n \sim 4-5$ for $\quad r>r_{s}$. However, this choice results in as much as $10 \%$ deviation in fulfilling the optical theorem. Thesedeviations are much larger than the sampling error $O\left(\left(\Delta r_{2}\right)^{2}\right)$ for finding the wave function. Such anerror in the phase of asymptotic wave function neverthelessresults in considerable deviation from theoptical theorem.

The importance of loss of accuracy can beexpressed in a one-dimensional example. Let us considera one-dimensional free equation for $\varphi=r \Psi$ and its finite difference approximation

$$
\begin{aligned}
\frac{d^{2}}{d r^{2}} \phi+k^{2} \phi(r) & =0 \rightarrow \frac{\phi(r+\Delta r)-2 \phi(r)+\phi(r-\Delta r)}{(\Delta r)^{2}}+ \\
+ & +k^{2} \phi(r)+O\left((\Delta r)^{2}\right)=0
\end{aligned} .
$$

Replacing the value of the function at points $\varphi(r \pm \Delta r)$ with a Taylor expansion, we obtain

$$
\frac{\partial^{2}}{\partial r^{2}} \varphi(r)+\frac{(\Delta r)^{2}}{12} \frac{\partial^{4}}{\partial r^{4}} \varphi(r)+k^{2} \varphi(r)+O\left((\Delta r)^{4}\right)=0
$$

The solutions to this equation have the form

$$
\exp \left(\frac{\sqrt{2 \sqrt{-3+\sqrt{9-3(\Delta r k)^{2}} r}}}{\Delta r}\right)
$$

Expanding the exponent in a series with respect to $\Delta r$, we obtain

$$
i k r+\frac{1}{24} i k r(\Delta r k)^{2}+O\left(\Delta r^{4}\right)
$$

Since this expression is in the exponent, the secondterm can be ignored only if it is small in comparison to unity $\left(\frac{1}{24} k r(\Delta r k)^{2}<<1\right)$. This inequality is certainlynot satisfied for very large $r$. For example, phase term $\frac{1}{24} k r(\Delta r k)^{2}=0.05543 r$ at $\Delta r=0.016, k=\sqrt{300}$, and becomes equal to 5.5 when $r=100$. The problem ofthe loss of 
accuracy is thus due to the incongruity ofthe exact asymptotic form and the asymptotic behaviorwhen solving the equation in finite differences. The same result can be obtained in general form.Let us find the solution to finite difference equation

$$
\begin{gathered}
\frac{\phi(r+\Delta r)-2 \phi(r)+\phi(r-\Delta r)}{(\Delta r)^{2}}+ \\
+k^{2} \phi(r)=0
\end{gathered}, \ldots
$$

by substituting function $\varphi=\exp \left(i k_{e f f} r\right)$ in it.

Thisleads to the equation for $k_{\text {eff }}$ :

$$
4 \sin ^{2}\left(\frac{k_{e f f} \Delta r}{2}\right)=k^{2}(\Delta r)^{2}
$$

The finite difference equation thus has an exponentialsolution with wave parameter $k_{\text {eff }}$ :

$$
k_{e f f}=2 \arcsin \left(\frac{k \Delta r}{2}\right) / \Delta r .
$$

Expanding $k_{\text {eff }}$ in a series with respect to $\Delta r$, we obtain

$$
\begin{aligned}
k_{\text {eff }} & =k+\frac{1}{24} k(k \Delta r)^{2}+\frac{3}{640} k(k \Delta r)^{4}+ \\
& +\frac{5}{7168} k(k \Delta r)^{6}+O\left((k \Delta r)^{8}\right),
\end{aligned}
$$

As expected, the first terms of the series coincidewith Eq. (2).In a similar manner, we can obtain the value of $k_{\text {eff }}$ for more complicated problems, e.g., for two-dimensionalSchrodinger equation (1), sampled usingNumerov's method to an accuracy of $O\left((\Delta r)^{4}\right)$. Wemust in this case solve the following finite difference equation

$$
\frac{\left(1+\frac{(k \Delta r)^{2}}{12}\right) \exp \left(i k_{e f f} \Delta r\right)-2\left(1+\frac{(k \Delta r)^{2}}{12}\right)+\left(1+\frac{(k \Delta r)^{2}}{12}\right) \exp \left(-i k_{e f f} \Delta r\right)}{(\Delta r)^{2}}+k^{2}=0, \ldots
$$

It yields

$$
k_{\text {eff }}=\frac{2 \arcsin \left(\frac{3 k \Delta r}{\sqrt{\left(36+3(k \Delta r)^{2}\right)}}\right)}{\Delta r},
$$

without quadratic terms with respect to $\Delta r$ as a series expansion

$$
k_{e f f}=k+\frac{1}{480} k^{5}(\Delta r)^{4}+O\left((\Delta r)^{6}\right)
$$

The deviation of the correct asymptotic behavior fromthe solution to Eq. (4) thus occurs at much greater distanceswhen using Numerov's scheme. For example, the value $\frac{1}{480} r_{a s} k(\Delta r k)^{4}$ at $r_{a s}=$ 100 is 0.02 , determiningan error of $2 \%$ in the unitarity condition whenusing the problem parameters $\Delta r=0.016, k=\sqrt{300}$. Such a scheme can be used for a two-dimensionalequation with twice differentiable interaction potentials.
Unfortunately, it does not work when using potentials with sharp boundaries. We propose introducing small term $-k^{2} \frac{(k \Delta r)^{2}}{12}$ into Eq. (3). On the one hand, this term has a low degree ofdiscretization error in the range of potentials; on theother, it is the compensating term for the free equationand brings its discretization error down to $O(\Delta r)^{4}$ :

$$
\begin{aligned}
\frac{d^{2}}{d r^{2}} \phi+k^{2} \phi(r) & =0 \rightarrow \frac{\phi(r+\Delta r)-2 \phi(r)+\phi(r-\Delta r)}{(\Delta r)^{2}}+ \\
+ & k^{2}\left(1-\frac{(k \Delta r)^{2}}{12}\right) \phi(r)=0 .
\end{aligned}
$$

In this case, we obtain for $k_{\text {eff }}$ the equation

$$
k_{e f f}=\frac{2 \arcsin \left(\frac{k \Delta r}{12} \sqrt{\left(36+3(k \Delta r)^{2}\right)}\right)}{\Delta r}
$$


whose series expansion

$$
k_{e f f}=k-\frac{1}{720} k^{5}(\Delta r)^{4}+O\left((\Delta r)^{6}\right),
$$

has a numerical factor in front of $(\Delta r)^{4}$ that is even less than in expansion (5), ensuring 99-97\% unitarity withthe most stringent parameters of the problem.

The computational error does not exceed $0.5 \%$ in testreductions of the sampling step (down to $\Delta r=0.008$ ).

\section{Results and discussions}

The problem of the scattering of a rigid diatomicmolecule on a thin disk was solved by using the correctasymptotic behavior. The barrier potential of a disk in cylindrical coordinates $\rho=r \cos \vartheta$ and $z=r \sin \vartheta$ is written in the form

$$
V_{b}=\left\{\begin{array}{cc}
V_{b 0}: & \rho \leq R \text { и }|z| \leq a / 2, \\
0: & \rho>R \text { или }|z|>a / 2 .
\end{array}\right.
$$

Parameters $V$, adetermine the disk size. In analogy with the problem of a rigid moleculepassing through a barrier [4], the potential in Eq. (1) for the model of a rigid molecule has the form

$$
V_{b}(\rho, z+d / 2)+V_{b}(\rho, z-d / 2),
$$

where parameter $d$ corresponds to the size of a scatteredrigid molecule.The difference between the scattering of a moleculeand a point particle equal to its mass is best seen inbackscattering, so not only the scattering cross sectionsof the molecule but also the backscattering cross section for a particle with a mass equal to that of twoatoms are shown in the figures below. This cross sectionmust have the form of monotonic energy and rapidlyshrink at energies above that of potential barrier(the disk potential). Barrier height $V_{b 0}$ in the abovecalculations was assumed to be $333.2 \AA^{-2}(4000 \mathrm{~K}$ fora hydrogen molecule). The figures show the total crosssections along with those into the front and rear hemispheres.The cross section into the front (rear) hemisphereis determined in the same way as the total crosssection, but the limits of integration over the angle arefrom 0 to $\pi / 2$ (from $\pi / 2$ to $\pi$ ).

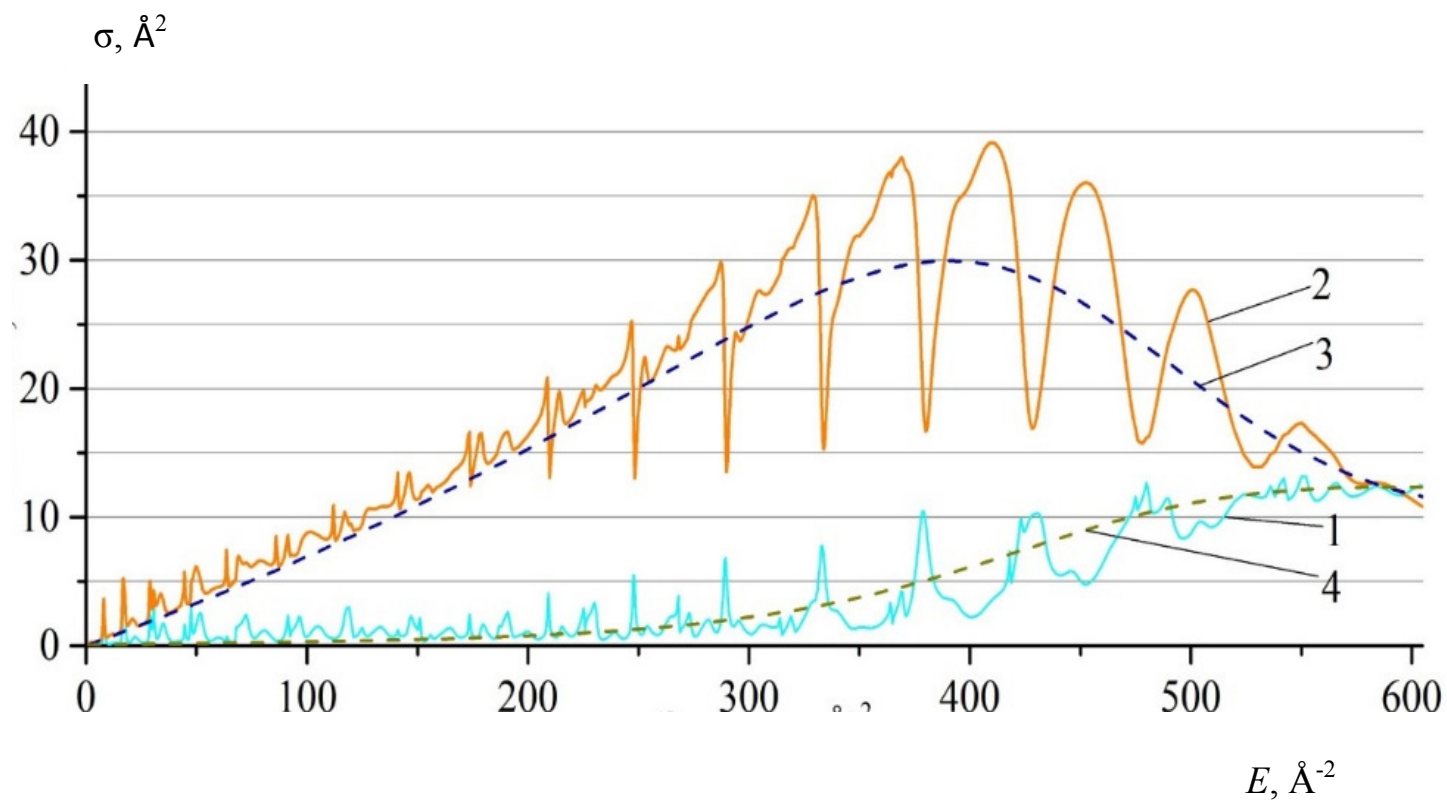

Figure 1 - The scattering cross sections of the hydrogen molecule on the disk at $\mathrm{R}=3 \AA .1-\sigma_{t}, 2-\sigma_{f}, 3-\sigma_{b}, 4$ - backward scattering cross section for a particle with a doubled hydrogen atom mass 
Figure 1 shows the results from calculating thedependence of the total scattering cross section of rigidhydrogen molecule $\mathrm{H}_{2}$ by a thin disk with a radius of $3 \AA$ and a width of $0.2 \AA$ on the energy. Parameter $d$ is $0.7416 \AA$ for hydrogen [10]. It can be seen that the backscatteringcross section of the molecule generallyrepeats the pattern of the scattering cross section for anunstructured particle, but it has characteristic resonance deviations.

The diffraction scattering cross sections of rigidberyllium molecule $\mathrm{Be}_{2}$ are given for comparison in Figure 2. Parameter $d$ is $2.47 \AA[11,12]$. The greater numberof quasi-bound states are due to the greater distancebetween atoms that is observed on the cross section by a large number of resonances. The main dips inthe cross section (resonances) coincide with the positionsof the energy levels in a rectangular one-dimensionalwell with a width of $d$, just as in the pattern forthe resonant passage of a rigid molecule [4]. This leadsto the differences between the scattering of hydrogenand beryllium molecules observed in the figures. Thenumber of minima in the total scattering cross sectionof a rigid beryllium molecule is much higher.

$$
\sigma, \AA^{2}
$$

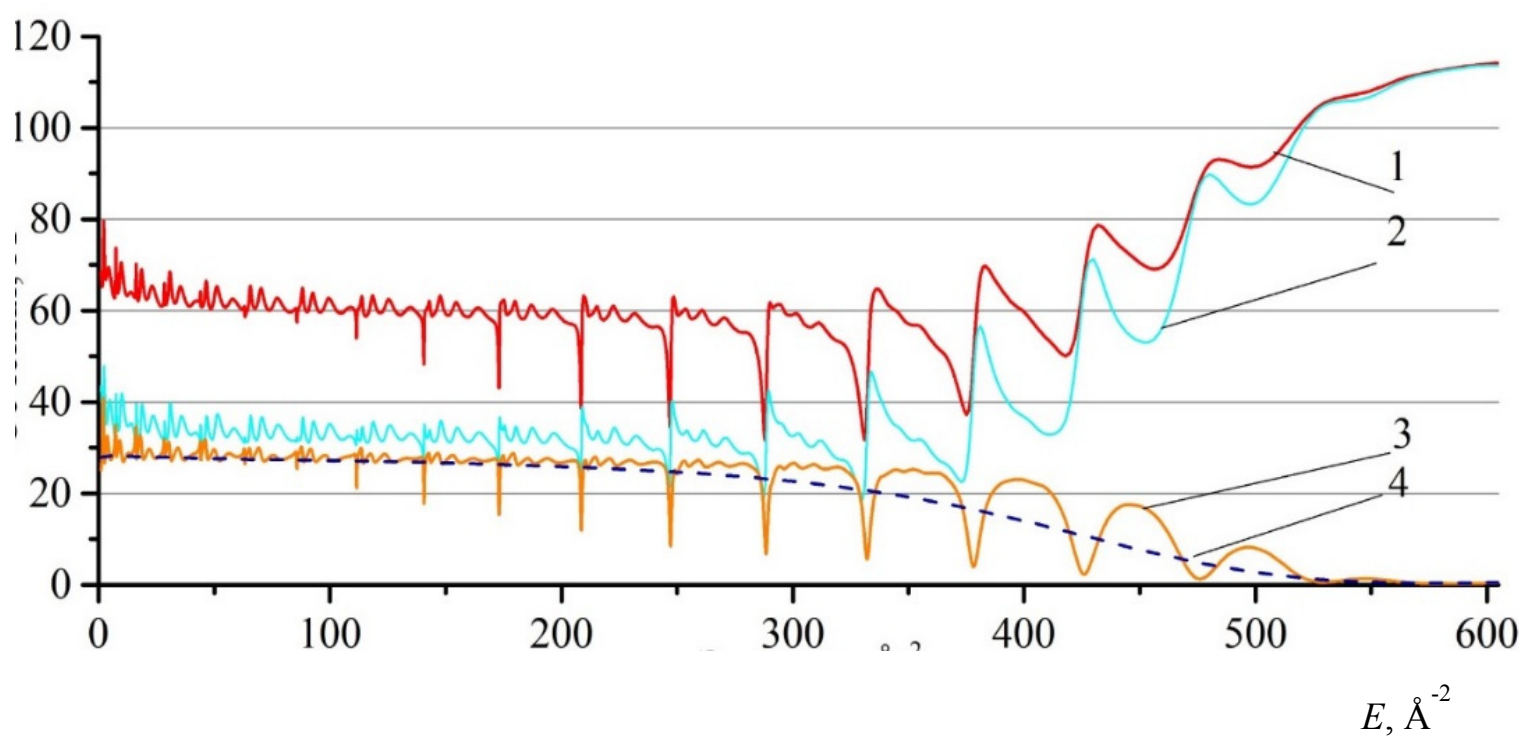

Figure 2 - The scattering cross sections of the Beryllium molecule on the disk at $\mathrm{R}=3 \AA$. Numerical line are liked one in Figure 1.

The calculation of two-dimension scattering at non-symmetrical center can be used for problem of scattering at holes in infinity wall. The
Babinet's principle [13] has been used for it. The numerical example is presented by Figure 3. 


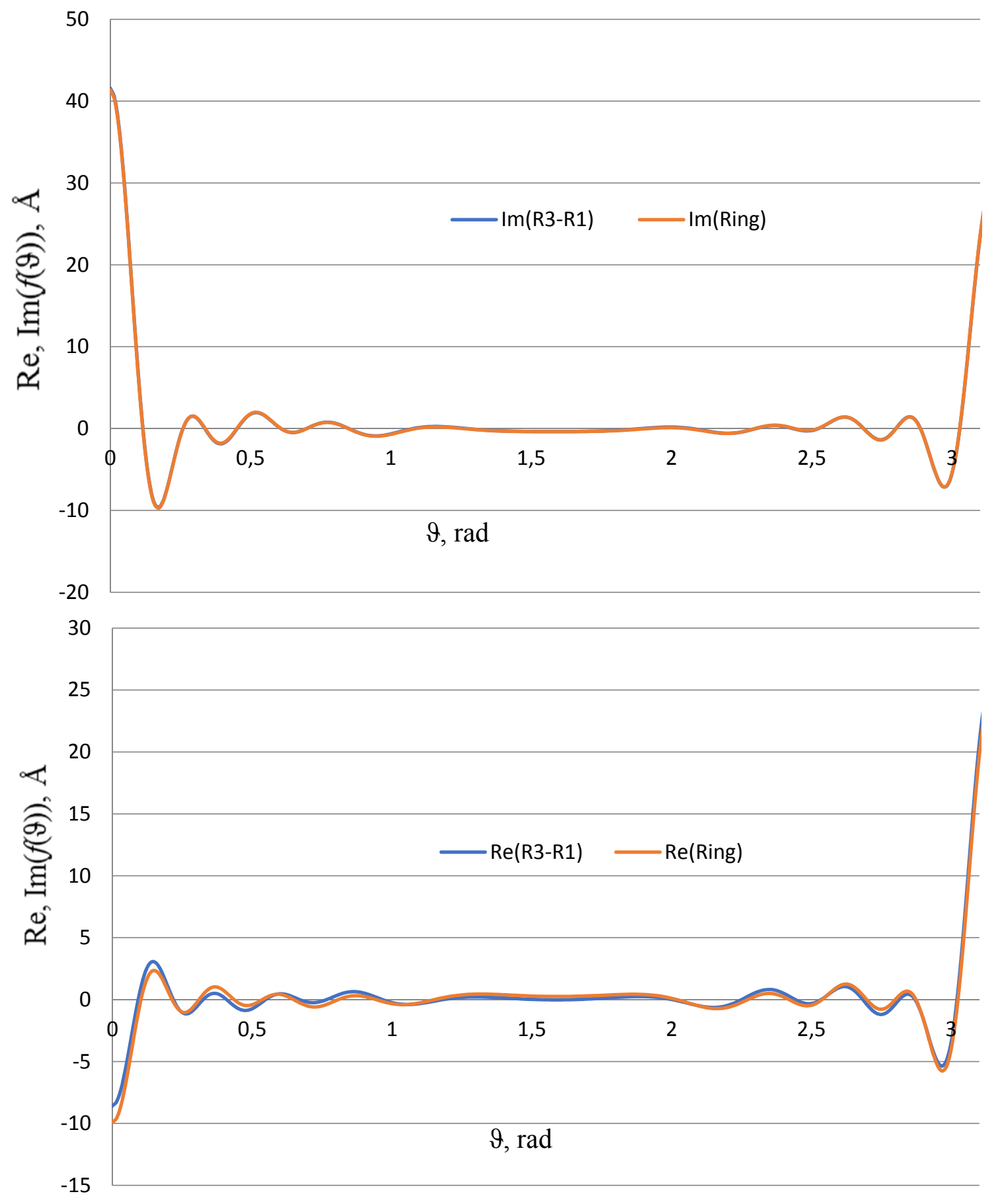

Figure 3 - Comparison of beryllium atom scattering at an energy of $100 \AA^{-2}$. R3 is the scattering on a disk of $3 \AA$, R1 is scattering on a disk of $1 \AA$, Ring is scattering on a ring $3 \AA$ wide with a hole of $1 \AA$.

It can be seem, that calculated scattering on the disk with big radius is equivalent a sum of scattering on ring with external radius which equal big disk's one and little disk with radius which equal hole's one.
The calculation of scattering on hole is presented at Figure 4.

It can seem that the resonance structure is exist here too. 


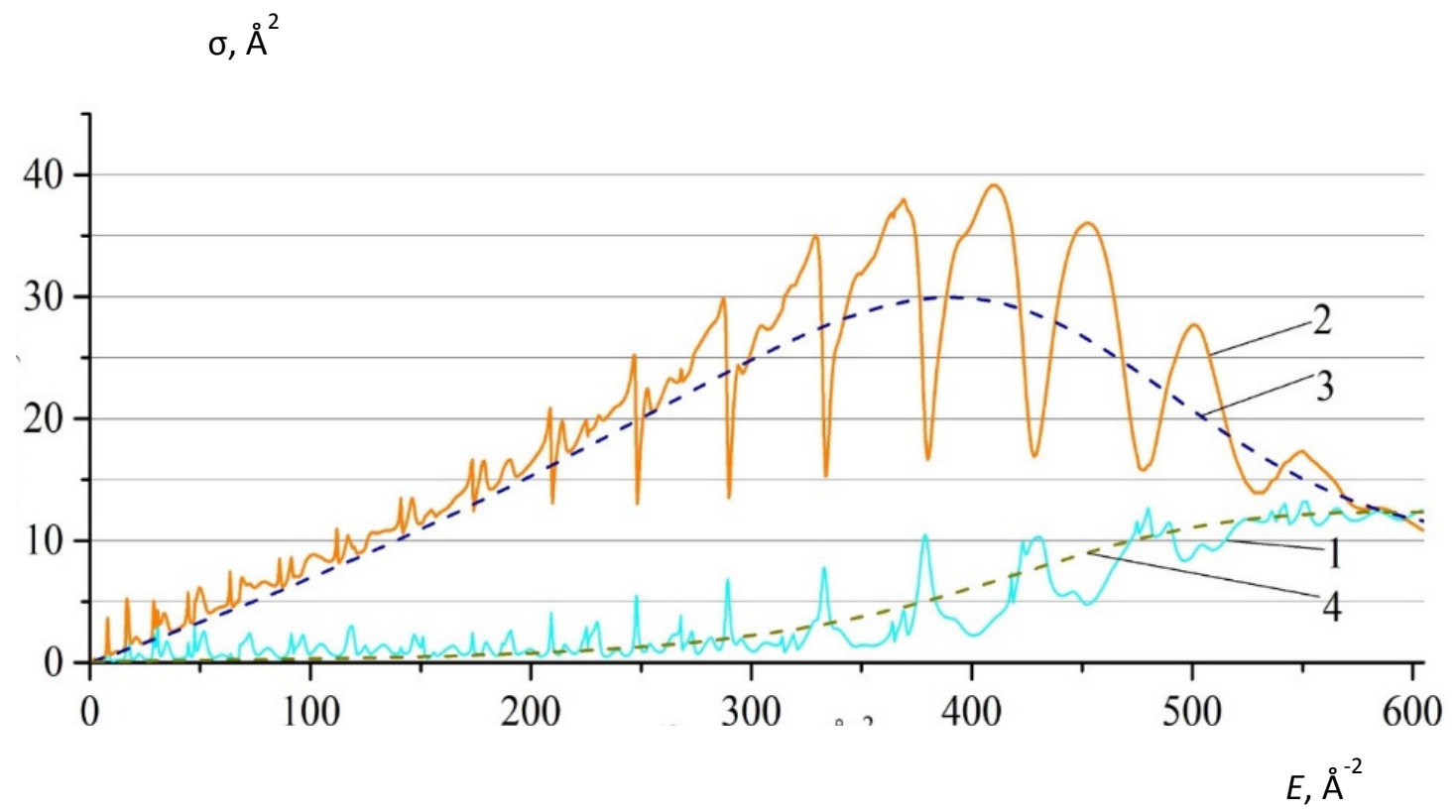

Figure 4 - Diffraction of beryllium on hole $\mathrm{R}=2 \AA$. Numerical line are liked one in Figure 1

\section{Conclusions}

The problem for scattering of rigid molecules bythe disks was successfully solved using an improvedsampling scheme that provides the correct asymptotic behavior. The result could be useful for analogical topics of investigation [14-22]. Corresponding diffraction scattering curves are of a pronounced resonance nature. The units of the problem length $\mathrm{E}$ were determinedby the interaction parameters. A femtometer (fm) isconveniently chosen as units of length in problems ofnuclear physics. The proposed way of correcting for thewave parameter in asymptotic behavior can thus beapplied in different areas that study diffraction using two-dimensional differential equations (e.g., in studyingparticle scattering by nonspherical atomic nuclei).

\section{References}

1. P. Krassovitskiy, F. Pen'kov. Quantum scattering by nonspherical objects. // Bull. Russ.Acad. Sci.: Phys.-2017. Vol. 81, - No. 6. - P. 730-734.

2. G. Satchler, Direct Nuclear Reactions. Oxford: Oxford Univ.Press, -1983.

3. P. Krassovitskiy, F. Pen'kov. Contribution of resonance tunneling of molecule to physical observables //J. Phys. B. - 2014. - Vol. 47. - No. 22. - P. 225210-225214.

4. R. Mathaes, et al. Non-spherical micro-and nanoparticles: fabrication, characterization and drug delivery applications. // ExpertOpin. Drug Delivery.-2015 - Vol. 12. - No.3. - P. 481-492.

5. A. Ikot, et al. Exact solutions of Schrodinger equation with improved ring-shaped non-spherical harmonic oscillator and Coulomb potential // Commun. Theor. Phys. - 2015. - Vol. 65. - No.5. - P. 569-574.

6. A. Statt, et al. Crystal nuclei in melts: a Monte Carlo simulation of a model for attractive colloids. Mol. Phys.2015. - Vol. 113. -No. 17-18. - P. 2556-2570.

7. A. Cronin, et al. Optics and interferometry with atoms and molecules. Rev. Mod. Phys. - 2009. -Vol. 81. -No. 3. - P. 1051-1129.

8. L. Landau, E. Lifshitz. Kvantovaya mekhanika (nerelyativistskaya teoriya) (Quantum Mechanics: NonRelativistic Theory), Moscow: Nauka, - 1989. - P. 583.

9. Spravochnik khimika (Chemistry Handbook), Moscow-Leningrad: GNTI Khim. Lit., - 1966, - Vol. 1. - P. 338. 
10. W. Jinlan, et al. Density functional study of beryllium clusters with gradient correction. // Phys.: Condens. Matter. - 2001. - Vol. 13. - P. L753-L758. 1548.

11. J. Merritt, et al. Beryllium Dimer - Caught in the act of bonding // Science. - 2009. - Vol. 324. - No. 5934. - P.

12. M. Born, E. Wolf. Principles of Optics. Cambridge: Cambridge University Press, - 1999.

14. A. Gusev, et al. Adiabatic representation for atomic dimers and trimers in collinear configuration // Physics of Atomic Nuclei. - 2018. - Vol. 81, - No 6. - P. 911-936.

15. A. Gusev, et al. Metastable states of a composite system tunneling through repulsive barriers // Theoretical and Mathematical Physics. -2016. - Vol. 186. - No.1.- P. 21-40.

16. O. Chuluunbaatar et al. Quantum transparency of barriers and reflection from wells for clusters of identical particles // Bull. Russ.Acad. Sci.: Phys. - 2018. - Vol. 82. - No. 6. - P. 648-653.

17. O. Chuluunbaatar et al. Solution of quantum mechanical problems using finite element method and parametric basis functions // Bull. Russ.Acad. Sci.: Phys. - 2018. - Vol. 82. - No. 6. - P. 654-660.

18. A. Gusev, et al. Resonant tunneling of a few-body cluster through repulsive barriers. // Physics of Atomic Nuclei. - 2014. - Vol. 77. - No. 3. - P. 389-413.

19. A. Gusev, et al. Parametric bases for elliptic boundary value problem // Journal of Physics Conference Series. 2018. - Vol. 965. - P. 012016-1-7.

20. A. Gusev, et al. Symbolic-numerical algorithms for solving elliptic boundary-value problems using multivariate simplex lagrange elements // Lecture Notes in Computer Science. - 2018. - Vol. 11077. - P. 197-213.

21. A. Gusev, et al. "Interpolation hermite polynomials for finite element method // EPJ Web of Conferences. 2018. - Vol. 173. - P.03009.

22. S. Vinitsky, et al. Three-body scattering model: diatomic homonuclear molecule and atom in collinear configuration // Proceedings of SPIE. - 2017. - Vol. 10337. - P. 103370j. 